\title{
Acupuncture versus Lornoxicam in the Treatment of Acute Renal Colic: A Randomized Controlled
} Trial

\author{
Xiaohua Zhang ${ }^{1, *}$ \\ Xinguo Liu $^{2, *}$ \\ Qiongxiang $\mathrm{Ye}^{2}$ \\ Xunbao Wang ${ }^{2}$ \\ Jinjun Chen ${ }^{2}$ \\ Zhiyong Wang ${ }^{2}$ \\ Pengfei Zhao ${ }^{2}$ \\ Baozhou Tao ${ }^{2}$ \\ Guoping $X u^{2}$ \\ Wanfeng $X u^{2}$ \\ Kan $\mathrm{Wu}^{2}$ \\ Yao $\mathrm{Xiao}^{3}$ \\ Li Yang' \\ Junqiang Tian' \\ Juan Wang' \\ Zhilong Dong' \\ Zhiping Wang (D)
}

'Institute of Urology, Lanzhou University Second Hospital; Key Laboratory of Gansu Province for Urological Diseases;

Gansu Nephro-Urological Clinical Center, Lanzhou, Gansu Province, People's Republic of China; ${ }^{2}$ Department of Urology, Susong County People's Hospital, Susong, Anhui Province, People's Republic of China; ${ }^{3}$ Department of Pediatrics, Lanzhou University Second Hospital, Lanzhou, Gansu Province, People's Republic of China

*These authors contributed equally to this work

Correspondence: Zhiping Wang

Email wangzplzu@I63.com
Objective: To compare the analgesic efficacy and safety of acupuncture and lornoxicam in acute renal colic (ARC).

Design, Setting, Participant: A randomized, double-blind, parallel-controlled, singlecentered trial was conducted at Susong County People's Hospital from October 2019 to November 2020. Eighty-four patients with ARC were randomly divided into lornoxicam group (Group L) and acupuncture group (Group A). Group A was treated with acupuncture at Sanyinjiao (SP6), Yinlingquan (SP9) and normal saline, and Group L was treated with sham acupuncture at SP6, SP9 and lornoxicam.

Main Outcome Measures: Visual analogue scale (VAS) scores and adverse reactions such as nausea and dizziness were recorded within 5, 10, 15, 20 and 40 minutes after treatment. The main outcome of this study was the short-term effective (STE) rate, the secondary outcome was the onset time, and the safety index was incidence of adverse reactions.

Results: A total of 80 patients completed this study, including 41 patients ( 21 males and 20 females) in Group L and 39 patients (21 males and 18 females) in Group A. Group A exhibited lower scores versus group $\mathrm{L}$ after treatment $(\mathrm{P}<0.05)$. The overall STE of group L was $61.00 \%(25 / 41)$, significantly lower than group A [84.62\% $(33 / 39)](\mathrm{P}<0.001)$. There was no difference in the incidence of adverse reactions between group A [2.6\% (1/39)] and group $\mathrm{L}[7.3 \%(3 / 41)](\mathrm{P}=0.616)$. The ordered logistic regression analysis showed patients receiving acupuncture therapy are more likely to be cured $[\mathrm{OR}=2.887,95 \% \mathrm{CI}$ : (1.190, 7.000), $\mathrm{P}=0.019]$.

Conclusion: Acupuncture at SP6, SP9 and intramuscular injection of lornoxicam can effectively and safely relieve ARC, but the former has faster and better analgesic effect. Moreover, the incidence of adverse reactions was similar between the two treatments. This acupuncture therapy is recommended as a complementary therapy for ARC.

Keywords: acupuncture, lornoxicam, renal colic, analgesia

\section{Introduction}

The worldwide incidence of kidney stones is 114 to 720 per 100,000, with a prevalence of $1.7 \%$ to $14.8 \%$, and this prevalence is increasing year by year. ${ }^{1}$ The ongoing challenge of growing members of kidney stone disease (KSD) has been proven to link to global warming, with a major component, rising temperature, contributing to a $24-84 \%$ increase in stone presentation as well as an increase of 1.6 to 2.2 million urolithiasis cases over a lifetime in the United States by $2050 .^{2} \mathrm{KSD}$ results in huge medical bills-for example, a total cost of £190-324 million in an acute or surgical treatment caused by upper tract stones in the UK, equivalent to the cost of prostate and 
bladder cancer combined in 2018. ${ }^{3}$ Acute renal colic (ARC) is a common complication of renal-ureteral calculi, ${ }^{4}$ causing $7-9 \%$ of all emergency department visits, excluding trauma patients. ${ }^{5}$ Pain relief is the primary goal with non-steroidal anti-inflammatory drugs (NSAIDs) recommended as firstline treatment for patients with an acute stone episode. ${ }^{4}$ Beyond that, adverse events of NSAIDs such as nausea, vomiting, and even allergic reactions, kidney failure, or gastrointestinal bleeding may offset the benefit-an utmost concern raised by health-care officials. ${ }^{6}$ In addition, opioids, alpha blockers, spasmodic drugs, and surgery are also recommended to treat $\mathrm{ARC},{ }^{4,7}$ but opioids drugs may have some adverse reactions and abuse problems, ${ }^{6,8}$, and surgery is very expensive. ${ }^{10}$

Previous studies have ascertained that acupuncture is effective in chronic pain relief, superior to placebos, ${ }^{11,12}$ with improved patient satisfaction, lower costs, and fewer adverse reactions. ${ }^{13}$ However, insufficient evidence from sparse data of acupuncture's effect on ARC lead to a growing demand for relevant RCTs. Therefore, we conducted a rigorous RCT that compared the efficacy and safety of acupuncture versus lornoxicam, an NSAID with analgesic property, in ARC. ${ }^{14,15}$

\section{Materials and Methods}

\section{Trails Design}

A randomized, double-blind, parallel-controlled, singlecentered trial was designed and conducted to assess the efficacy and safety of acupuncture versus lornoxicam in ARC treatment. Patients diagnosed as ARC at our hospital from October 2019 to November 2020 were recruited. The study protocol was consistent with the Declaration of Helsinki and has been approved by the Ethics Committee of Susong County People's Hospital (Ethics Number: 2019-001A) and registered at Chinese Clinical Trial Registry (http://www.chictr.org.cn), the registration ID is ChiCTR1900026517. We have the patient's written consent that any information should be kept as anonymous as possible.

\section{Drugs and Acupuncture Needle Involved in the Trail}

1. Lornoxicam for injection, drug specifications: $8 \mathrm{mg} /$ ampoule, Yangtze River Pharmaceutical Group Co., LTD. National Medicine Permission Number (NMPN): H20080329; 2. 0.9\% Normal saline for injection, drug specification: $100 \mathrm{~mL} /$ bottle, CR Double-Crane Pharmaceutical
Co., LTD. National Medicine Permission Number (NMPN): H61020015; 3. Aseptic acupuncture needle, specification 0.16mm*100mm, Taixing Tianhe Medical Instrument Co., LTD. Producing Certificate of Jiangsu Province Food \& Drug Administration: No. 2013-0073.

\section{Participants}

Inclusion Criteria

Patients were included in our study if they (i) were male or female of ureteral calculi confirmed by ultrasonography, KUB (kidney-ureter-bladder), or CT (computed tomography), (ii) aged 18 to 75 years, (iii) had no prior or ongoing history of analgesics use before the visit, (iv) had a visual analog scale (VAS) score of $\geq 3$ at baseline; (v) wounds, ulcers, varicose veins, and other factors affecting the efficacy evaluation were undetectable at the acupoints. Patients signed the informed consent form before entering the study.

\section{Exclusion Criteria}

Patients were excluded if they fulfilled the following criteria: (i) ureteral stricture or deformity; (ii) renal colic caused by a steinstrasse after extracorporeal shock wave lithotripsy; (iii) unstable vital signs, for example, septic shock; (iv) unable to cooperate with treatment due to mental illness or cognitive impairment; (v) pregnant and menstruating women; (vi) allergic to NSAIDs; (vii) active gastrointestinal ulcer/bleeding; (viii) needle sickness, coagulation and fibrinolysis disorder, long-term anticoagulant, sedative, analgesic, or antidepressant therapy; (ix) other conditions unsuitable for an entry into the study, as defined by the researcher.

\section{Interventions}

\section{Acupoint Selection and Operation Specification}

The name and location of acupoints in this study were referenced to the Nomenclature and Location of Acupuncture Points described in the national GB/ T12346-2006 acupoint standard. Acupuncture procedures were implemented per GB/T21709.20-2009 Standardized Manipulations of Acupuncture and Moxibustion - Part 20: Basic Techniques of Filiform Needle. Two acupoints, Sanyinjiao (three yin intersection, SP6) and Yinlingquan (yin mound spring, SP9) were selected for acute pain relief in patients with ARC. SP6 is located on the medial side of the lower leg, 3 cun (cun means "body cun" or "fingerbreadth cun" or "proportional unit of body". Patients put the index finger, middle finger, ring finger and little finger together, take the striation of the second knuckle of the 
middle finger as the reference line, and the width of the four fingers as 3 cun.) superior to the prominence of the medial malleolus in a depression close to the medial crest of the tibia. SP9 is located on the medial side of the lower leg, at an angle formed by the medial condyle of the tibia and the posterior border of the tibia.

\section{Treatments}

Participants in the control (lornoxicam group, group L) and the treatment groups (acupuncture group, group A) received lornoxicam $(8 \mathrm{mg})$ and isovolume normal saline injections into the gluteus maximus, respectively. Acupoints were covered by self-adhesive skin electrodes after disinfection for blinding. The converted electrodes not only play a role in shielding, but also play a role in fixing acupuncture needles for sham acupuncture, which appears to be real acupuncture. For the patients in group A, needles were vertically inserted into bilateral SP6 and SP9 acupoints, up to 1 cun under the skin, and the acupuncturist manipulated the needles until patients had intensive deqi feeling (sour, numb, swelling up, or painful). For group L, the acupuncturist only needed to touch skin with the tip of the needle to make patients feel acupuncture sensation without penetrating their skin (Figure 1). After 20 min of needle retention, a standard bandage was applied to each acupoint for all patients in both groups.

\section{Outcomes}

The primary outcome of this trial included short-term outcomes (STE). The secondary outcomes were onset time and the frequency of adverse events.

\section{STE}

The VAS scores were assessed at the outset of acupuncture therapy and $40 \mathrm{~min}$ after the treatment. Patients self-reported the pain intensity score on a $10-\mathrm{cm}$ scale from 0 (painless) to 10 (unbearable pain). The pain relief rate (PRR) was calculated as follows: $P R R=\left(\frac{V A S_{0 \min }-V A S_{40 \min }}{V A S_{0 \min }}\right) \times 100 \%$.

Based on PRR values, therapeutic efficacy was graded into four levels: grades A (cure), B (markedly effective), $\mathrm{C}$ (effective), and D (ineffective). Grade A (PRR $=100 \%)$

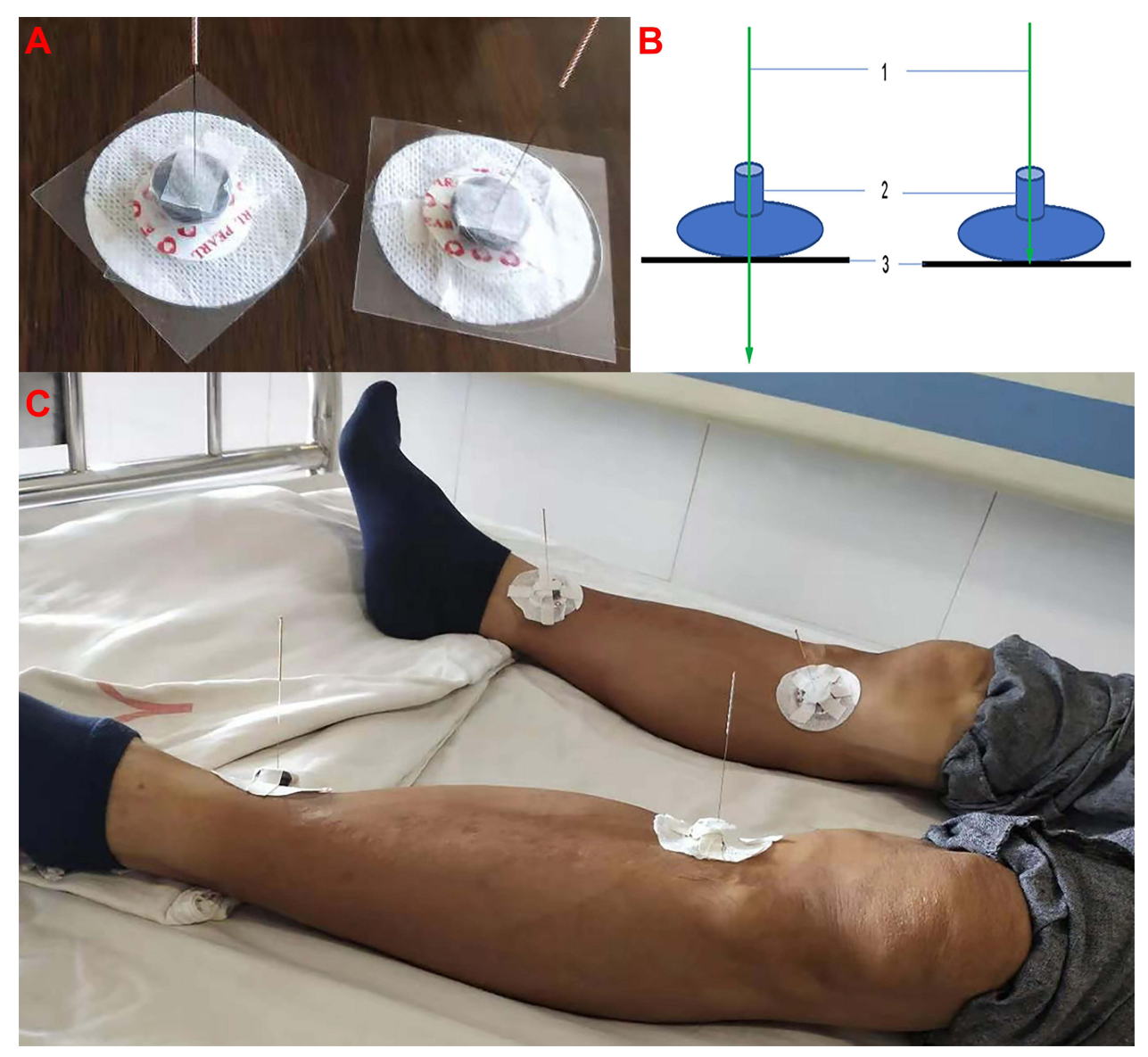

Figure I Schematic diagram of acupuncture and sham acupuncture. (A) sham acupuncture model; (B) conceptual diagram of acupuncture and sham acupuncture, I: needle; 2: self-adhesive medical electrode; 3: skin; (C) Model case. 
was defined as the complete disappearance of pain in the kidney or ureter after $40 \mathrm{~min}$ of acupuncture therapy. Grades B $(75 \% \leq \mathrm{PRR}<100 \%)$ referred to significant pain relief after the treatment. Grade $\mathrm{C}(50 \% \leq \mathrm{PRR}<75 \%)$ was rated in a case of pain improvement following acupuncture treatment. Patients reporting a nonsignificant amelioration were classified into grade D (PRR $<50 \%)$. STE was evaluated by the ratio of patients with satisfactory results (grades A, B, and C) to the total participants.

\section{Onset Time}

The time to onset was indirectly reflected by counting the number of patients with satisfactory results (grades A, B, or C) at $0-5,6-10,11-15,16-20,21-40$ min intervals and the number of cases without efficacy response after more than 40 min of treatment.

\section{Safety Evaluation}

All adverse reactions reported during the trial were recorded by a specially trained nurse. The safety of acupuncture was assessed by the frequency of adverse events.

\section{Sample Size}

The response rate of lornoxicam for $\mathrm{ARC}$ was $75 \%,{ }^{15}$ and the response rate of acupuncture was estimated to be $90 \%$. Our significance level was $\alpha=0.05$, and the power of the trial was $0.1(\beta), \delta$ was defined as a difference in the response rate between groups $\mathrm{A}$ and $\mathrm{L}$.

The sample size was calculated using the following formula:

$$
\begin{gathered}
\mathrm{N}=\pi_{0}\left(1-\pi_{0}\right)\left[\frac{u_{\alpha}+\mu_{\beta}}{\delta}\right]^{2} \\
\pi_{0}=75 \%, \pi_{1}=90 \% \delta=\pi_{1}-\pi_{0}=0.15 \%, \mu_{0.1}=1.282, \mu_{0.05}= \\
1.645, \mathrm{~N}=71.3 \approx 72
\end{gathered}
$$

Concerning a drop-out rate of no more than $20 \%$, only 84 patients (42 in each group) were ultimately included in our study.

\section{Randomization, Allocation Concealment and Blinding}

All participants were stratified by sex and randomly assigned to either group by a blind, independent statistician. Random numbers were generated with RV. UNIFORM generator in SPSS 25.0 and mapped to predefined number ranges (1-42 for female patients and 4384 for male patients). All random numbers were allocated to group $\mathrm{A}$ and $\mathrm{L}$ by using Visual Binning instruction according to the number size, with 21 male and 21 female patients in each group. Then the statistician made a random number table with three lines. The first line is the sequence number of enrolled patients, the second line is the generated random number that corresponded to the sequence number, and the third line is the grouping identification, with " $\mathrm{A}$ " represents the acupuncture group and "L" represents the lornoxicam group. Then the statistician made enrollment cards with random numbers and treatment methods and sequentially concealed in opaque envelopes marked 1-84, to an out-patient physician for patient recruitment. Female or male patients who meet the inclusion criteria were randomly selected from envelopes numbered 1-42 or numbered 43-84, respectively. Eligible participants were asked to send their envelopes to the acupuncturist who determined the treatment based on the number in the envelope.

Patients were blinded to the treatment plan. The performing acupuncturist was neither blinded to the treatment nor allowed to participate in the follow-up and statistical analysis. The nurse who recorded VAS scores was also blinded to the treatment. Acupuncture procedures were manipulated by the same acupuncturist, followed by the assessment by an independent nurse to avoid bias.

\section{Statistical Methods}

Continuous variables following normal distribution were expressed as mean \pm standard deviation (SD), while those with a non-normal distribution as median (IQR). Independent-sample $T$-test or Mann-Whitney $U$-test was employed to compare the differences between two groups, and the rank-sum test was used to compare data before and after the treatment in each group. Categorical variables were described as frequencies (or percentages), and differences between groups were analyzed using the chi-square test or Wilcoxon test. All data were processed using SPSS version 25.0 (SPSS Inc., Chicago, IL, USA).

\section{Results}

Initially, $143 \mathrm{ARC}$ patients were recruited in this study, and 59 were excluded according to the eligibility criteria, of whom 34 declined participation, 17 did not fulfill the inclusion criteria, and 8 had a history of analgesic use before the visit. There were 84 participants eligible for analysis. During the study, two females in group A and one in group L dropped out as they discontinued VAS assessments. Another female with an intense fear of 
acupuncture manipulation was ruled out from group A. Finally, 39 patients (21 males and 18 females) in group $\mathrm{A}$ and 41 (21 males and 20 females) in group $\mathrm{L}$ were included (Figure 2).

\section{Comparison of Clinical Characteristics Between the Acupuncture versus Lornoxicam Groups}

There were no statistically significant differences in gender, age, body temperature, pulse rate, systolic blood pressure (SBP), stone size and location, and the degree of hydronephrosis between groups A and L $(P>0.05)$ (Table 1A). However, diastolic blood pressure (DBP) in group L was markedly higher than that in group A $(P=0.041)$. This might be attributed to the limited statistical power caused by the small sample size, without impacts on final results.

Thirty-seven cases $(46.25 \%)$ presented with calculi in the left ureter and $43(53.75 \%)$ had right ureter stones. Cases of lower ureteric stones occupied 58.75\% (47/80) of all participants, followed by the upper ureteric stone $(23 /$ $80,28.75 \%)$ and the middle ureteric stone $(10 / 80$,
$12.50 \%)$. Despite five cases $(6.25 \%)$ with no hydronephrosis, different degrees of hydronephrosis were detectable in most participants at admission. Among them, 85\% (68/80) exhibited mild hydronephrosis, 8.75\% (7/80) moderate hydronephrosis, and severe hydronephrosis was undetectable (Table 1B and C).

Details of underlying conditions of 9 cases $(23.08 \%)$ in group A and 7 (17.07\%) in group L incorporated hypertension, diabetes, coronary heart disease, and others. Ureteric and renal stones confirmed by either CT scan or ultrasonography were found in 16 patients (41.03\%) in group A and 11 $(26.83 \%)$ in group L. There were 3 patients combined with renal cysts and one with bladder stones in group L, 2 with renal cysts and 3 with teratoma, pleural effusion, and hepatic cysts, respectively, in group A (Supplementary Table 1).

\section{Correlation Analysis of VAS Score on Admission}

Multiple linear regression was used to analyze the correlation between admission VAS score and gender, stone location, stone size and degree of hydronephrosis. The results showed that taking lower ureteral calculi, no hydronephrosis

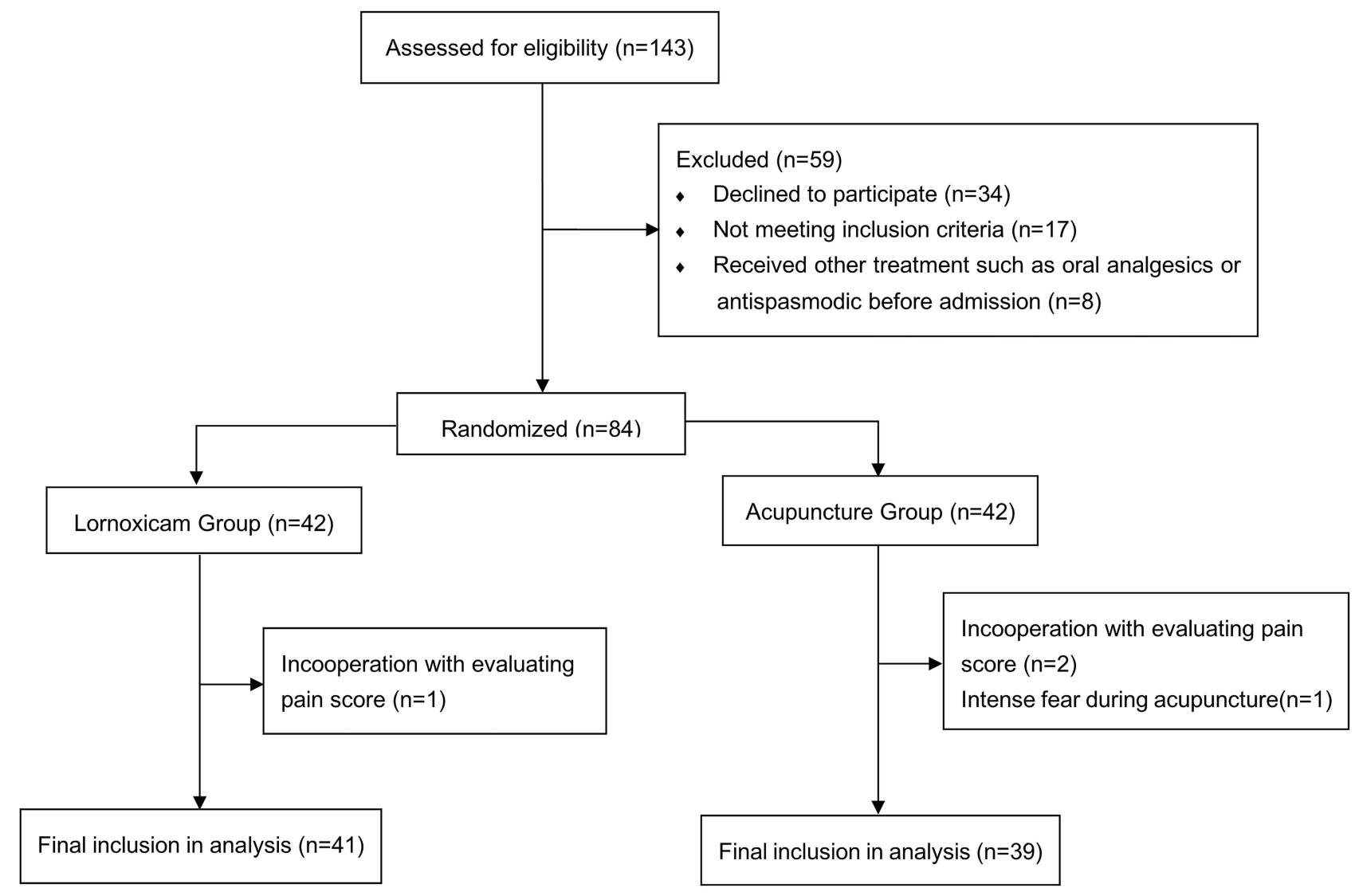

Figure 2 Patient enrolment flow chart. 
Table I Baseline Characteristics of Both Groups

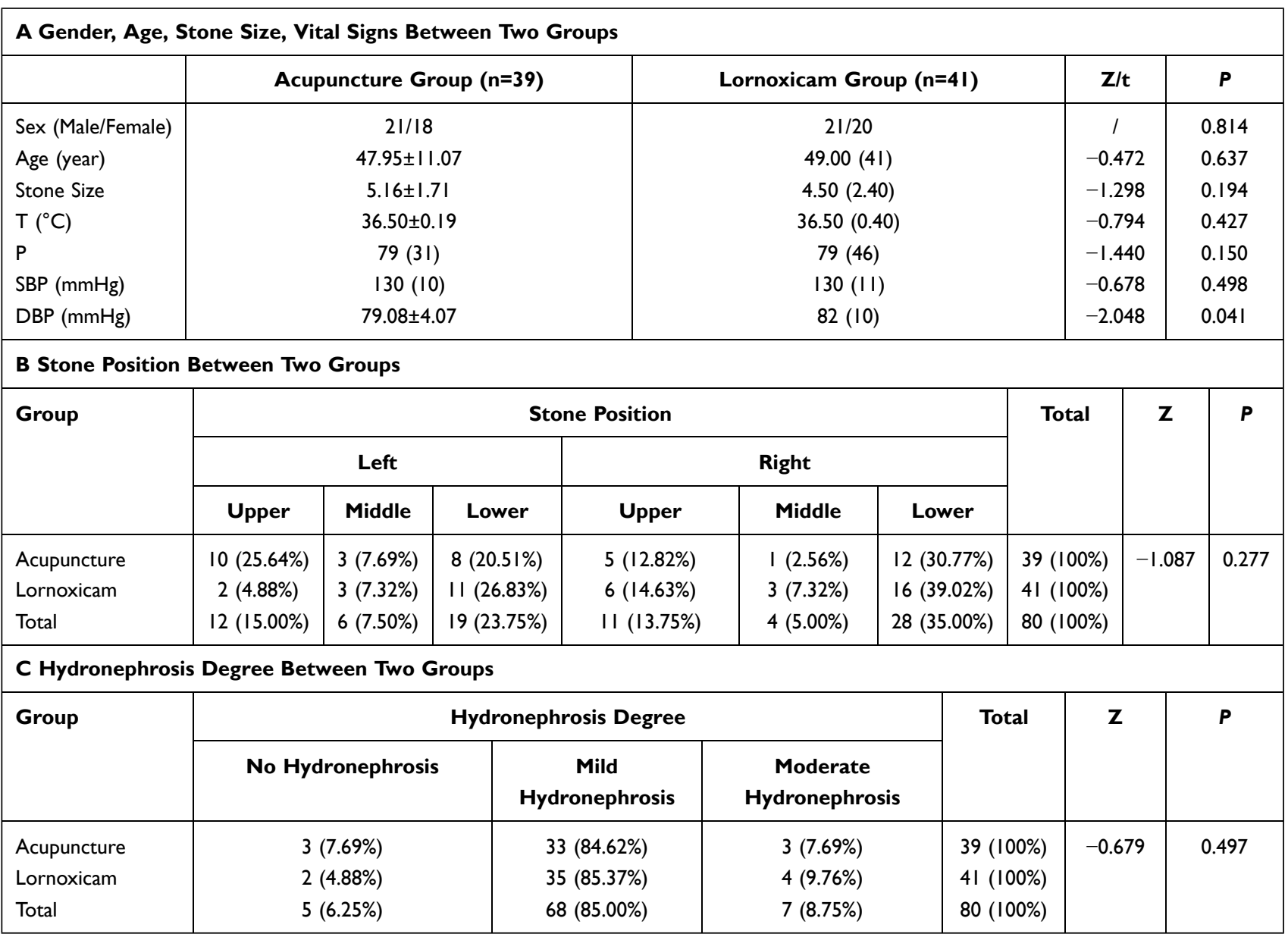

and female patients as reference, upper or middle ureteral calculi, mild or moderate hydronephrosis, male patients had no effect on admission VAS score $(P>0.05)$, and stone size had no effect on admission VAS score $(P>0.05)$ (Table 2$)$.

\section{Lower VAS Scores After Acupuncture Therapy}

The baseline VAS scores were the same between the two groups $(P=0.957)$. Participants in groups $\mathrm{A}$ and L reported significantly lower VAS scores at 5, 10, 15, 20 , and $40 \mathrm{~min}$ post-treatment versus baseline scores $(P<0.001)$. Group A exhibited lower scores versus group $\mathrm{L}$ at $5,10,15,20$, and $40 \mathrm{~min}$ post-treatment $(P<0.05)$ (Table 3 and Figure 3).

\section{STE is Improved After Acupuncture}

\section{Therapy}

According to the 4-grade outcome assessment, 7 cases $(17.95 \%)$ were rated as grade A, $21(53.85 \%)$ were grade B, 5 (12.82\%) were grade C, and 6 (15.38\%) were grade $\mathrm{D}$ in the group $\mathrm{A}$. Therefore, the overall STE of acupuncture was $84.62 \%$ (33/39). However, only 2 cases (4.88\%) were graded as "cure", 19 $(46.34 \%)$ as "markedly effective", $4(9.76 \%)$ as "effective", and $16(39.02 \%)$ as "ineffective" in group L. The overall STE of lornoxicam was $61.00 \%$ (25/41), significantly lower than group A $(P=0.000)$. The grading of each group was summarized in Table 4 .

\section{Faster Onset of Pain Relief Provided by}

\section{Acupuncture}

As a result, $89.70 \%(35 / 39)$ and $92.30 \%$ (36/39) of patients in group A responded within 15 and $40 \mathrm{~min}$, respectively, versus $21.95 \%$ (9/41) and 60.98\% (25/41) in group L. There were statistically significant differences between the two groups $(P=0.000)$, indicating a faster onset of pain relief delivered by acupuncture (Table 5). 
Table 2 Correlation Analysis of VAS Score on Admission with Gender, Stone Size and Hydronephrosis Degree

\begin{tabular}{|c|c|c|c|c|c|c|c|}
\hline \multirow[t]{2}{*}{ Parameter } & \multirow{2}{*}{$\frac{\text { Standardized Coefficients }}{\text { B }}$} & \multirow[t]{2}{*}{$\mathbf{t}$} & \multirow[t]{2}{*}{$\mathbf{P}$} & \multicolumn{2}{|c|}{$95.0 \% \mathrm{Cl}$ for $\mathrm{B}$} & \multicolumn{2}{|c|}{ Collinearity Statistics } \\
\hline & & & & & & Tolerance & VIF \\
\hline (Constant) & & 18.697 & 0.000 & 7.179 & 8.893 & & \\
\hline Stone size & -0.012 & -0.089 & 0.929 & -0.097 & 0.088 & 0.716 & 1.397 \\
\hline \multicolumn{8}{|l|}{ Upper ureter (Ref.) } \\
\hline Lower ureter & -0.040 & -0.291 & 0.772 & -0.425 & 0.317 & 0.672 & 1.488 \\
\hline Middle ureter & -0.223 & -1.604 & 0.113 & -0.991 & 0.107 & 0.669 & 1.495 \\
\hline \multicolumn{8}{|l|}{ No hydronephrosis (Ref.) } \\
\hline Moderate hydronephrosis & 0.266 & $1.47 \mid$ & 0.146 & -0.219 & 1.452 & 0.396 & 2.526 \\
\hline Mild hydronephrosis & 0.263 & 1.540 & 0.128 & -0.142 & 1.109 & 0.442 & 2.261 \\
\hline \multicolumn{8}{|l|}{ Male (Ref.) } \\
\hline Female & 0.035 & 0.290 & 0.772 & -0.267 & 0.358 & 0.913 & 1.095 \\
\hline
\end{tabular}

Table 3 VAS Score Difference Between the Two Groups After Treatment

\begin{tabular}{|l|c|c|c|c|c|c|}
\hline Group & VAS $_{\mathbf{0 m i n}}$ & VAS $_{\mathbf{5 m i n}}$ & VAS $_{\mathbf{1 0 m i n}}$ & VAS $_{\mathbf{1 5 m i n}}$ & VAS $_{\mathbf{2 0 m i n}}$ & VAS $_{\mathbf{4 0 m i n}}$ \\
\hline Acupuncture & $8.00(1.2)$ & $3.00(3)^{\mathrm{a}}$ & $3.00(2)^{\mathrm{a}}$ & $2.00(2)^{\mathrm{a}}$ & $2.00(2)^{\mathrm{a}}$ & $2.00(2)^{\mathrm{a}}$ \\
Lornoxicam & $8.10(0.9)$ & $7.00(1)^{\mathrm{a}}$ & $7.00(2.3)^{\mathrm{a}}$ & $6.00(2)^{\mathrm{a}}$ & $5.00(4)^{\mathrm{a}}$ & $2.00(5)^{\mathrm{a}}$ \\
Z & -0.054 & -5.714 & -5.899 & -5.486 & -4.411 & -2.232 \\
$P$ & 0.957 & $<0.001$ & $<0.001$ & $<0.001$ & $<0.001$ & 0.026 \\
\hline
\end{tabular}

Note: a $P<0.05$ (Compare VAS at different time points in the same group, $Z$ is not given).

\section{Related Factors on the Efficacy of Acupuncture}

The effect grade was taken as dependent variable, and the treatment method, stone size and stone location were taken as independent variables for ordered Logistic regression analysis. The Omnibus test result shows the model was meaningful $(P=0.048)$. The results indicated the size of the stone $[\mathrm{OR}=0.529,95 \% \mathrm{CI}:(0.191,1.463), P=0.529]$ and the location of the stone [comparison between upper and lower ureter: OR $=1.918,95 \% \mathrm{CI}:(0.707,5.206), P=0.201$, comparison between middle and lower ureter: $\mathrm{OR}=2.219,95 \% \mathrm{CI}$ : (0.556,8.858), $P=0.259)]$ were no significant correlation between acupuncture efficacy. The treatment method is the only factor affecting the short-term effective rate of patients. Patients receiving acupuncture therapy had a greater chance of being cured $[\mathrm{OR}=2.887,95 \% \mathrm{CI}$ : $(1.190,7.000), P=0.019]$ (Supplementary Table 2).

\section{Safety Comparison Between Acupuncture versus Lornoxicam}

Adverse reactions in group $\mathrm{L}$ consisted of nausea (2 cases) and dizziness (1 case) post-treatment, which completely disappeared after bed rest. One patient in group A reported frequent urination after acupuncture, whose condition was spontaneously relieved 2 hours later. The frequency of adverse reactions was $2.6 \%$ in group $\mathrm{A}$ and $7.3 \%$ in group L. There was a nonsignificant difference between the two groups $(P=0.616)$.

\section{Outcomes of Patients in Group A and Group L}

In group A, 6 patients (15.38\%) had spontaneous stone expulsion, 10 patients $(25.64 \%)$ were discharged after conservative treatment, and 23 patients $(58.97 \%)$ received extracorporeal shock wave lithotripsy, ureteral stents, or transurethral ureteroscopic lithotripsy. In the group L, there were $1(2.44 \%), 8$ (19.51\%) and 32 $(78.05 \%)$ patients with these three outcomes, respectively. There was no statistically significant difference between the two groups $(P=0.074)$ (Supplementary Table 3). 


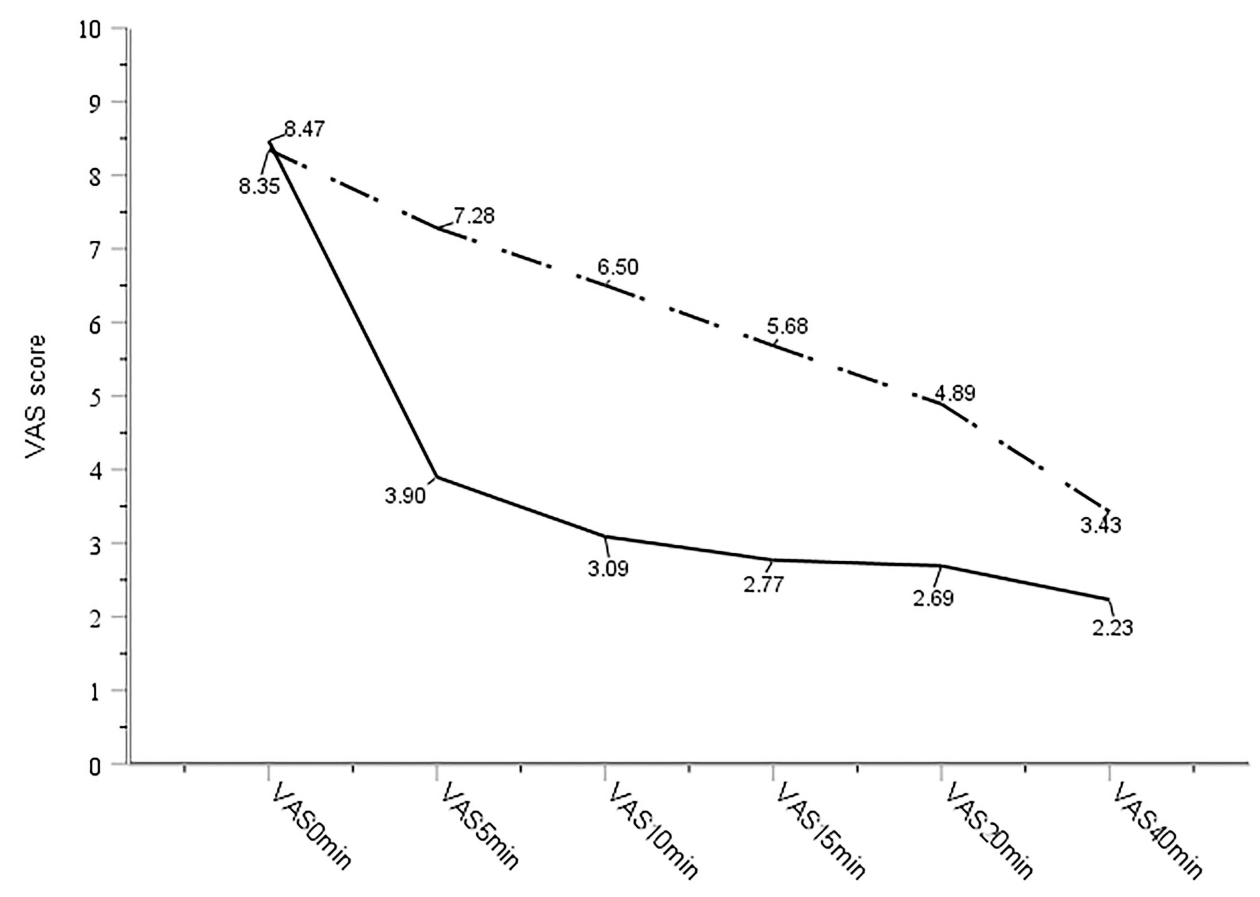

Figure 3 VAS scores of the two groups after treatment.

Notes: - Acupuncture group. $\longrightarrow$ Lornoxicam group.

\section{Discussion}

\section{Research Design Innovation}

Acupuncture is a unique form of traditional Chinese medicine that has been ubiquitously used as a well-tolerated and effective source for pain relief, and numerous acupuncture studies have been published since the 1970s. ${ }^{16}$ However, the efficacy of acupuncture in the treatment of ARC is currently lacking in evidence-based medical evidence, mainly due to the lack of high-quality randomized controlled clinical trials. First, there is a lack of exact description of random method, allocation concealment and blinding method in study design. Secondly, the types and methods of use of the control drugs are not recommended in the guidelines. For example, intravenous infusion of morphine was used as the control group in the recent study of Beltaief et al, ${ }^{17}$ while Chen and $\mathrm{Li}^{18}$ chose intramuscular injection of Fortanodyn as the control group.
Third, there were few trials with placebo acupuncture, which will lead to different psychological expectations of patients due to different treatment, and may also lead to bias in VAS score during follow-up. Finally, the selection of complex acupoints and the manipulation of acupuncture at specific points require a long period of training, which limits the promotion of acupuncture therapy. ${ }^{17-19}$ In our study, acupuncture offered a rapid, robust analgesic effect in ARC, superior to lornoxicam. Manipulations at SP6 and SP9 in the lower legs were an easy job without pronounced adverse reactions. All these unfold the feasibility of acupuncture for acute pain. In addition, intramuscular administration of lornoxicam was used as a positive control in this study for the following three reasons: (1) EAU (European Association Of Urology) Guidelines recommend NSAIDs as the first choice for ARC; ${ }^{4}$ (2) There is no difference in the efficacy of intramuscular

Table 4 Comparison of Short-Term Efficacy Between the Two Groups

\begin{tabular}{|c|c|c|c|c|c|c|c|}
\hline \multirow[t]{2}{*}{ Group } & \multicolumn{4}{|c|}{ Grade } & \multirow[t]{2}{*}{ Total } & \multirow[t]{2}{*}{$\mathbf{Z}$} & \multirow[t]{2}{*}{$\mathbf{P}$} \\
\hline & A (Cured) & B (Markedly Effective) & C (Effective) & D (Ineffective) & & & \\
\hline Acupuncture & 7 (I7.95\%) & $21(53.85 \%)$ & $5(12.82 \%)$ & $6(15.38 \%)$ & $39(100 \%)$ & -3.903 & $<0.001$ \\
\hline Lornoxicam & $2(4.88 \%)$ & 19 (46.34\%) & $4(9.76 \%)$ & $16(39.02 \%)$ & $41(100 \%)$ & & \\
\hline Total & 9 (II.25\%) & $40(50.00 \%)$ & 9 (II.25\%) & $22(27.5 \%)$ & $80(100 \%)$ & & \\
\hline
\end{tabular}


Table 5 Comparison of Onset Time Between the Two Groups

\begin{tabular}{|c|c|c|c|c|c|c|c|c|c|}
\hline \multirow[t]{2}{*}{ Group } & \multicolumn{6}{|c|}{ Onset Time } & \multirow[t]{2}{*}{ Total } & \multirow{2}{*}{$\begin{array}{l}\text { Likelihood } \\
\text { Ratio }\end{array}$} & \multirow[t]{2}{*}{$P$} \\
\hline & $0-5 \mathrm{~min}$ & $6-10 \mathrm{~min}$ & $11-15 \mathrm{~min}$ & $16-20 \mathrm{~min}$ & $21-40 \mathrm{~min}$ & $>40 \mathrm{~min}$ & & & \\
\hline Acupuncture & 22 (56.4I\%) & 10 (25.64\%) & 3 (7.69\%) & $0(0.00 \%)$ & I (2.56\%) & 3 (7.69\%) & $39(100 \%)$ & 65.574 & $<0.001$ \\
\hline Lornoxicam & $0(0.00 \%)$ & $2(4.88 \%)$ & 7 (I7.07\%) & 10 (24.39\%) & 7 (I7.07\%) & 15 (36.59\%) & $4 \mid(100 \%)$ & & \\
\hline Total & 22 (27.50\%) & 12 (I5.00\%) & 10 (12.50\%) & 10 (12.50\%) & $8(10.00 \%)$ & I8 (22.50\%) & $80(100 \%)$ & & \\
\hline
\end{tabular}

administration of lornoxicam and intravenous administration of lornoxicam for ARC. ${ }^{14}$ Third, intramuscular injection of NSAIDs in the treatment of ARC can have more lasting analgesic effect and fewer adverse reactions. ${ }^{20}$ Finally, the ideal sham acupuncture must meet two main criteria: on the one hand, there is no or only a small specific effect, on the other hand, there is no difference or high similarity between other aspects, allowing the successful implementation of blind method. ${ }^{21}$ A meta-analysis has shown that non-penetrating acupuncture at nonacupoints or acupoints are more suitable for sham acupuncture. ${ }^{11}$ In order to avoid different mental expectations of patients for different therapies, we chose nonpenetrating acupuncture at acupoints as sham acupuncture therapy.

\section{Efficacy and Safety of Treatment}

Acute renal colic is one of the most severe pain. Sasmaz and Kirpat found that the pain level was independent of stone size. ${ }^{22}$ In addition, $11 \%$ of patients had no hydronephrosis at the time of acute presentation, and $71 \%$ presented with mild hydronephrosis, ${ }^{23}$ only severe hydronephrosis was significantly related to stone size. ${ }^{24}$ In our study, $6.25 \%$ of patients had no hydronephrosis, $85 \%$ had mild hydronephrosis, and no patients with severe hydronephrosis. Our multiple linear regression analysis indicated that the VAS score at admission was independent of stone size, hydronephrosis degree and gender, which was generally consistent with the conclusion of previous studies.

In this study, the VAS score of patients receiving acupuncture at all time points was lower than that of the control group, and the final short-term effective rate was also higher than that of the control group. Orderly logistics analysis indicated that the treatment method was the only factor affecting the efficacy, and the probability of patients receiving acupuncture being cured was about 2.9 times higher than that receiving lornoxicam. In addition, the acupuncture effect was significantly faster than that of lornoxicam and could be rapidly activated within 10 minutes. This may be due to the following reasons: on the one hand, acupuncture analgesia may exert analgesic effect through opioid system and non-opioid system, which is a complex and rapid neurohumoral immune reaction. ${ }^{25}$ On the other hand, lornoxicam reduces prostaglandin synthesis via inhibiting cyclo-oxygenase 2 (COX-2) to exert an analgesic effect, and increases in plasma dynorphin and $\beta$ endorphin levels to inhibit spinal pain sensation will only be possible through at least 5 days of lornoxicam administration. $^{26}$ Therefore, acupuncture is superior to lornoxicam in relieving pain. All these indicate that acupuncture of SP6 and SP9 is a rapid and effective treatment for ARC.

The time of spontaneous expulsion of ureteral calculi was about 17 days, ${ }^{27}$ and the probability of spontaneous expulsion of calculi of different sizes and positions was different. The spontaneous expulsion rates of calculi $<5 \mathrm{~mm}, 5-7 \mathrm{~mm}$ and $>7 \mathrm{~mm}$ were $89 \%, 49 \%$ and $29 \%$, respectively. The spontaneous removal rates of calculi in upper, middle and lower ureteral segments were 52\%, 70\% and $83 \%$, respectively. ${ }^{28}$ In this study, six patients in group A had spontaneous stone expulsion after treatment, while only one in group L. Although there was no statistical difference $(P=0.074)$, it seems that acupuncture at SP6 and SP9 can promote the expulsion of ureteral stones, which requires further study.

It has been reported that about $1 \%$ of patients experience adverse reactions due to receiving analgesics when treating acute renal colic. ${ }^{27}$ However, adverse reactions caused by acupuncture are extremely rare, and the estimated probability of serious adverse reactions (such as nausea, syncope, and strong emotional reactions) is only $1.3 \%{ }^{29}$ In this study, both lornoxicam and acupuncture showed good safety. Although three patients in the group L had adverse reactions of nausea and dizziness, these adverse reactions were very mild and resolved spontaneously without any intervention. Moreover, one patient in group A reported frequent urination after acupuncture, and this symptom 
was relieved 2 hours later. We speculate that this condition is related to enhanced ureteral and bladder contraction or stone removal by acupuncture stimulation. Although there are no direct reports that acupuncture at SP6 and SP9 can promote ureteral contraction, Yi et al reported that acupuncture at SP6, Shuidao (ST28), Epangxian III (MS4) and Zusanli (ST36) can improve the maximal cystometric capacity, maximal flow rate, and bladder compliance of patients after total hysterectomy. ${ }^{30}$ Animal experiment has also shown that acupuncture at Weizhong (BL40), SP6 and SP9 can increase the intravesical pressure and bladder adenosine triphosphate (ATP) content in rabbits with acute urinary retention. ${ }^{31}$ Finally, it should be noted that acupuncture of SP6 is prohibited for pregnant women, because it is important to note that acupuncture is contraindicated for pregnant women because SP6 stimulation may also lead to miscarriage. $^{32}$

\section{Potential Mechanisms of Treatment Effectiveness}

Despite dilatation, extension, and spastic activity of ureter in acute ureteric calculus obstruction, molecular research has revealed enhanced local release of inflammatory mediators, such as prostaglanin F2 $\alpha$ (PGF2 $\alpha$ ) and 5-hydroxytryptamine (5-HT), and bradykinin synthesis, subsequently increasing renal intrapelvic pressure, resulting in severe and unbearable renal colic. ${ }^{33-35}$ Although myogenic factors are essential in the control of ureteral peristalsis, sympathetic and parasympathetic nerves also play a role in this process. Ureteral contraction can be enhanced by via activating $\alpha_{1}$-adrenergic, muscarinic, purinergic, histamine $\mathrm{H} 1$ receptors on ureteric smooth muscle cells as well as increased substance P, 5-HT levels cohesively, and the ureter is dilated through activating $\beta 2 / 3$-receptors and histamine $\mathrm{H} 2$ receptors and upregulating endothelial oxide and prostaglandin E1, E2 expressions. ${ }^{35}$ These mechanisms may be both the cause of renal colic and the effect of acupuncture.

The acupuncture analgesia mechanism is associated with nerve activity, body-fluid exchange, and endocrine regulation. ${ }^{25}$ Preexisting evidence shows that acupuncture manipulation can mitigate pain via promoting mast cell (MC) degranulation and ATP/adenosine, IL-10, and opioid peptide release, stimulating P2XRs (purinergic ligandgated cationic channels) and A1R (adenosine A1 receptor), and mechanical sensitive membrane channels such as ASIC3 (acid-sensing ion channel 3) and TRPV (transient receptor potential vanilloid 1), and downregulating pronociceptive receptor expression in peripheral neurons. ${ }^{25,36}$ Glial cell activation, resulting from signaling activation in the spinal cord by acupuncture manipulations, can be inhibited by downregulating the chemokine CX3CL1 (XC chemokine ligand) expression and upregulating the expression of anti-inflammatory IL-10. Glial cell inhibition may further reduce the release of tumor necrosis factor (TNF)- $\alpha$, IL-1 $\beta$, IL-6, prostaglandins E2, and other pain-related substances. ${ }^{37}$ In terms of the roles of the central nervous system, acupuncture provides analgesic relief via activating the periaqueductal gray (PAG), anterior cingulate cortex (ACC), rostral ventromedial medulla (RVM), and hippocampus to release $\beta$-endorphins and other central analgesic substances. ${ }^{25,37,38}$ Studies have shown that acupuncture of Sanyinjiao and Zusanli can reduce the expression of cyclooxygane-2 (COX-2) and the synthesis of prostaglandin E2 (PGE2) in spinal cord in animal models of nerve pain, and its analgesic effect is significantly better than celecoxib. ${ }^{39}$ Acupuncture at Hegu point and Sanyinjiao can relieve labor pain by increasing the expression of $\kappa$-opioid receptor and Prodynorphin protein in spinal cord, and thereby increasing the concentration of serum Dynorphin. ${ }^{40}$

Intersegmental neuronal connectivity is a determinant of specific acupoint-organ correlation. ${ }^{41}$ SP9 and SP6 are paired-acupoints for pain relief in urinary obstruction, including ARC, through dredging the meridians. ${ }^{42,43}$ Anatomically, ureter receives branches from $\mathrm{T}_{11}-\mathrm{L}_{2}$ and $\mathrm{S}_{2-4}$ nerve fibers, ${ }^{44}$ and branches from $\mathrm{L}_{4}-\mathrm{S}_{1}$ nerves are located in SP6 acupoint. ${ }^{45}$ Branches around SP9 are innervated by the tibial nerve. Therefore, it is speculated that SP6 and SP9 stimulation exert a potent analgesic effect via signal transduction from the tibial nerve to the corresponding spinal cord segments and brain regions.

\section{Limitations of This Study}

Our study has the following limitations. It was a singlecenter study with small sample size and insufficient objective measurements like serum or urinary substance P levels, due to limited funds. The 40-min observation is not long enough to examine the maintenance of efficacy of acupuncture and may underestimate the therapeutic effect of lornoxicam. Moreover, it is well known that there is no optimal type of sham acupuncture design. ${ }^{21}$ Therefore, although we had made some efforts in the blind method, it is not a recognized method and needs to be verified by more samples. Our sham acupuncture runs the risk of 
being identified by patients because it does not penetrate the skin. Furthermore, administration of normal saline to gluteus maximus as a control for lornoxicam remains to be improved as we found some patients to feel pain in injection area, although it was bearable. Finally, whether acupuncture can reduce hospitalization rates, costs and hospitalization days remains to be further assessed.

\section{Conclusion}

We found that acupuncture at SP6 and SP9 delivers a better therapeutic effect on ARC versus lornoxicam, which is worthy of recommendation in emergencies as such, particularly when analgesics do not work or patients are allergic to these drugs. The underlying stone passage and pro-peristaltic function of acupuncture at SP6 and SP9 need further validations.

\section{Acknowledgments}

We would like to thank all the members for their contributions to the success of this trial.

\section{Funding}

This research did not receive any specific grant from funding agencies in the public, commercial, or not-forprofit sectors.

\section{Disclosure}

Data Access and Responsibility: Our research data is open and transparent and available at http://www.chictr.org.cn/ showproj.aspx?proj=44279 or downloaded directly from $\mathrm{M}$ e g a ( h t t p s : / / m e g a.n z / fi 1 e / kZ9QmC4C\#zZvkbuSeUNBGXCG53IM1FpxHj7pPzwZa SOsCNZj-yeg) (https://pan.baidu.com/s/ 1cRdaGxsxyuGYK_OM5osaNQ, Password: 3apz). The principal investigator, Wang Zhiping, had full access to all of the data in the study and takes responsibility for the integrity of the data and the accuracy of the data analysis. The authors report no conflicts of interest in this work.

\section{References}

1. Khan SR, Pearle MS, Robertson WG, et al. Kidney stones. Nat Rev Dis Primers. 2016;2:16008. doi:10.1038/nrdp.2016.8

2. Geraghty RM, Proietti S, Traxer O, et al. Worldwide impact of warmer seasons on the incidence of renal colic and kidney stone disease: evidence from a systematic review of literature. J Endourol. 2017;31 (8):729-735. doi:10.1089/end.2017.0123

3. Geraghty RM, Cook P, Walker V, et al. Evaluation of the economic burden of kidney stone disease in the UK: a retrospective cohort study with a mean follow-up of 19 years. BJU Int. 2020;125(4):586-594. doi:10.1111/bju.14991
4. Turk C, Petrik A, Sarica K, et al. EAU guidelines on diagnosis and conservative management of urolithiasis. Eur Urol. 2016;69 (3):468-474. doi:10.1016/j.eururo.2015.07.040

5. Kaynar M, Koyuncu F, Buldu I, et al. Comparison of the efficacy of diclofenac, acupuncture, and Acetaminophen in the treatment of renal colic. Am J Emerg Med. 2015;33(6):749-753. doi:10.1016/j. ajem.2015.02.033

6. Pathan SA, Mitra B, Cameron PA. A systematic review and meta-analysis comparing the efficacy of nonsteroidal anti-inflammatory drugs, opioids, and paracetamol in the treatment of acute renal colic. Eur Urol. 2018;73(4):583-595. doi:10.1016/j. eururo.2017.11.001

7. Turk C, Petrik A, Sarica K, et al. EAU guidelines on interventional treatment for urolithiasis. Eur Urol. 2016;69(3):475-482. doi:10.1016/j.eururo.2015.07.041

8. Motov S, Drapkin J, Butt M, et al. Analgesic administration for patients with renal colic in the emergency department before and after implementation of an opioid reduction initiative. West $J$ Emerg Med. 2018;19(6):1028-1035. doi:10.5811/westjem.2018.9.38875

9. Hosier GW, McGregor T, Beiko D, et al. Increased risk of new persistent opioid use in pediatric and young adult patients with kidney stones. Can Urol Assoc J. 2020;14(8):237-244. doi:10.5489/cuaj.6796

10. Hollingsworth JM, Norton EC, Kaufman SR, et al. Medical expulsive therapy versus early endoscopic stone removal for acute renal colic: an instrumental variable analysis. J Urol. 2013;190(3):882-887. doi:10.1016/j.juro.2013.03.040

11. Vickers AJ, Vertosick EA, Lewith G, et al. Acupuncture for chronic pain: update of an individual patient data meta-analysis. $J$ Pain. 2018;19(5):455-474. doi:10.1016/j.jpain.2017.11.005

12. Xiang A, Cheng K, Shen X, et al. The immediate analgesic effect of acupuncture for pain: a systematic review and meta-analysis. Evid Based Complement Alternat Med. 2017;2017:3837194.

13. Jan AL, Aldridge ES, Rogers IR, et al. Review article: does acupuncture have a role in providing analgesia in the emergency setting? A systematic review and meta-analysis. Emerg Med Australas. 2017;29(5):490-498. doi:10.1111/1742-6723.12832

14. Soylu A, Sarier M, Altunoluk B, et al. Comparison of the efficacy of intravenous and intramuscular lornoxicam for the initial treatment of acute renal colic: a randomized clinical trial. Urol J. 2019;16 (1):16-20. doi:10.22037/uj.v0i0.4496

15. Cevik E, Cinar O, Salman N, et al. Comparing the efficacy of intravenous tenoxicam, lornoxicam, and dexketoprofen trometamol for the treatment of renal colic. Am J Emerg Med. 2012;30 (8):1486-1490. doi:10.1016/j.ajem.2011.12.010

16. Fan AY, Miller DW, Bolash B, et al. Acupuncture's role in solving the opioid epidemic: evidence, cost-effectiveness, and care availability for acupuncture as a primary, non-pharmacologic method for pain relief and management-white paper 2017. J Integr Med. 2017;15 (6):411-425. doi:10.1016/S2095-4964(17)60378-9

17. Beltaief K, Grissa MH, Msolli MA, et al. Acupuncture versus titrated morphine in acute renal colic: a randomized controlled trial. $J$ Pain Res. 2018;11:335-341. doi:10.2147/JPR.S136299

18. Chen M, Li S-M. Clinical observation of the efficacy on renal colic treated with electroacupuncture at the Xi-cleft points of kidney and bladder meridians as well as the relevant Front-mu points. World $J$ Acupunct Mox. 2012;22(1):7-12. doi:10.1016/S1003-5257(12)60002-6

19. Lee YH, Lee WC, Chen MT, et al. Acupuncture in the treatment of renal colic. J Urol. 1992;147(1):16-18. doi:10.1016/S0022-5347(17)37121-5

20. Pathan SA, Mitra B, Straney LD, et al. Delivering safe and effective analgesia for management of renal colic in the emergency department: a double-blind, multigroup, randomised controlled trial. Lancet. 2016;387 (10032):1999-2007. doi:10.1016/S0140-6736(16)00652-8

21. Yuan QL, Wang P, Liu L, et al. Acupuncture for musculoskeletal pain: a meta-analysis and meta-regression of sham-controlled randomized clinical trials. Sci Rep. 2016;6:30675. doi:10.1038/srep30675 
22. Sasmaz M, Kirpat V. The relationship between the severity of pain and stone size, hydronephrosis and laboratory parameters in renal colic attack. Am J Emerg Med. 2019;37(11):2107-2110. doi:10.1016/ j.ajem.2019.06.013

23. Song Y, Hernandez N, Gee MS, et al. Can ureteral stones cause pain without causing hydronephrosis? World J Urol. 2016;34 (9):1285-1288.

24. Hiller N, Berkovitz N, Lubashevsky N, et al. The relationship between ureteral stone characteristics and secondary signs in renal colic. Clin Imaging. 2012;36(6):768-772. doi:10.1016/j. clinimag.2012.01.018

25. Li Y, Yang M, Wu F, et al. Mechanism of electroacupuncture on inflammatory pain: neural-immune-endocrine interactions. J Trad Chin Med. 2019;39(5):740-749.

26. Balfour JA, Fitton A, Barradell LB. Lornoxicam. A review of its pharmacology and therapeutic potential in the management of painful and inflammatory conditions. Drugs. 1996;51(4):639-657. doi:10.2165/00003495-199651040-00008

27. Yallappa S, Amer T, Jones P, et al. Natural history of conservatively managed ureteral stones: analysis of 6600 patients. J Endourol. 2018;32(5):371-379. doi:10.1089/end.2017.0848

28. Shah TT, Gao C, Peters M, et al. Factors associated with spontaneous stone passage in a contemporary cohort of patients presenting with acute ureteric colic: results from the Multi-centre cohort study evaluating the role of Inflammatory Markers In patients presenting with acute ureteric Colic (MIMIC) study. BJU Int. 2019;124(3):504-513. doi:10.1111/bju.14777

29. Kelly RB, Willis J. Acupuncture for pain. Am Fam Physician. 2019;100(2):89-96.

30. Yi WM, Chen Q, Liu CH, et al. Acupuncture for preventing complications after radical hysterectomy: a randomized controlled clinical trial. Evid Based Complement Alternat Med. 2014;2014:802134. doi:10.1155/2014/802134

31. Chen LL, Wu CY, Sui MH. Effects of electroacupuncture of "Weizhong"(BL 40),"Sanyinjiao"(SP 6) and "Yinlingquan"(SP 9) on intravesical pressure and bladder adenosine triphosphate content in rabbits with acute urinary retention. Acupunct Res. 2012;37 (4):291-295.

32. Mollart LJ, Adam J, Foureur M. Impact of acupressure on onset of labour and labour duration: a systematic review. Women Birth. 2015;28(3):199-206. doi:10.1016/j.wombi.2015.03.007

33. Kapila V, Kapila AK, Tailly T, et al. The analgesic action of desmopressin in renal colic. Acta Clin Belg. 2017;72(3):179-185. doi:10.1080/17843286.2016.1230569
34. Jankovic SM, Stojadinovic D, Stojadinovic M, et al. Angiotensin receptor blocker losartan inhibits spontaneous motility of isolated human ureter. Eur J Drug Metab Pharmacokinet. 2016;41 (6):835-838. doi:10.1007/s13318-015-0298-x

35. Canda AE, Turna B, Cinar GM, et al. Physiology and pharmacology of the human ureter: basis for current and future treatments. Urol Int. 2007;78(4):289-298. doi:10.1159/000100830

36. He JR, Yu SG, Tang Y, et al. Purinergic signaling as a basis of acupuncture-induced analgesia. Purinergic Signal. 2020;16 (3):297-304. doi:10.1007/s11302-020-09708-z

37. Chen T, Zhang WW, Chu YX, et al. Acupuncture for pain management: molecular mechanisms of action. Am J Chin Med. 2020;48 (4):793-811. doi:10.1142/S0192415X20500408

38. Lv Q, Wu F, Gan X, et al. The involvement of descending pain inhibitory system in electroacupuncture-induced analgesia. Front Integr Neurosci. 2019;13:38. doi:10.3389/fnint.2019.00038

39. Lau WK, Lau YM, Zhang HQ, et al. Electroacupuncture versus celecoxib for neuropathic pain in rat SNL model. Neuroscience. 2010;170(2):655-661. doi:10.1016/j.neuroscience.2010.07.031

40. Jiang QY, Wang MY, Li L, et al. Electroacupuncture relieves labour pain and influences the spinal dynorphin/א-opioid receptor system in rats. Acupunct Med. 2016;34(3):223-228. doi:10.1136/acupmed2015-010951

41. Chen SZ. An analysis on correlation between acupoint and target organs of acupuncture-moxibustion from the perspective of modern medical sciences. Acupunct Res. 2019;44(8):620-624. doi:10.13702/ j.1000-0607.180575

42. Wee TC, Tan YL. Observations regarding battlefield acupuncture to treat low back pain in the emergency department? Am J Emerg Med. 2019;37(1):151. doi:10.1016/j.ajem.2018.04.065

43. Deadman P, Al-Khafaji M, Baker KA. Manual of Acupuncture. 2nd ed. England: Journal of Chinese Medicine Publications; 2001.

44. Frober R. Surgical anatomy of the ureter. BJU Int. 2007;100 (4):949-965. doi:10.1111/j.1464-410X.2007.07207.x

45. Ma YX, Ma LX, Liu XL, et al. A comparative study on the immediate effects of electroacupuncture at Sanyinjiao (SP6), Xuanzhong (GB39) and a non-Meridian point, on menstrual pain and uterine arterial blood flow, in primary dysmenorrhea patients. Pain Med. 2010;11(10):1564-1575. doi:10.1111/j.1526-4637.2010.00949.x
Journal of Pain Research

\section{Publish your work in this journal}

The Journal of Pain Research is an international, peer reviewed, open access, online journal that welcomes laboratory and clinical findings in the fields of pain research and the prevention and management of pain. Original research, reviews, symposium reports, hypothesis formation and commentaries are all considered for publication. The manuscript management system is completely online and includes a very quick and fair peer-review system, which is all easy to use. Visit http:// www.dovepress.com/testimonials.php to read real quotes from published authors. 